\title{
Hemofiltration compared to hemodialysis for acute kidney injury: systematic review and meta-analysis
}

Jan O Friedrich ${ }^{1,2,3,4,5^{*}}$, Ron Wald ${ }^{1,2,4}$, Sean M Bagshaw ${ }^{6}$, Karen EA Burns ${ }^{1,2,3,4,5}$ and Neill KJ Adhikari ${ }^{1,5,7}$

\begin{abstract}
Introduction: The objective of this systematic review and meta-analysis was to determine the effect of renal replacement therapy (RRT), delivered as hemofiltration vs. hemodialysis, on clinical outcomes in patients with acute kidney injury (AKI).

Methods: MEDLINE, EMBASE and CENTRAL databases and conference abstracts were searched to June 2012 for parallel-group or crossover randomized and quasi-randomized controlled trials (RCTs) evaluating hemofiltration vs. hemodialysis in patients with AKI. Two authors independently selected studies and abstracted data on study quality and outcomes. Additional information was obtained from trial authors. We pooled data using randomeffects models.

Results: Of 6,657 citations, 19 RCTs (10 parallel-group and 9 crossover) met inclusion criteria. Sixteen trials used continuous RRT. Study quality was variable. The primary analysis included three parallel-group trials comparing similar doses of hemofiltration and hemodialysis; sensitivity analyses included trials comparing combined hemofiltration-hemodialysis or dissimilar doses. We found no effect of hemofiltration on mortality (risk ratio (RR) $0.96,95 \%$ confidence interval (Cl) 0.73 to $1.25, P=0.76$; three trials, $\mathrm{n}=121$ (primary analysis); $\mathrm{RR} 1.10,95 \% \mathrm{Cl} 0.88$ to $1.38, P=0.38$; eight trials, $\mathrm{n}=540$ (sensitivity analysis)) or other clinical outcomes (RRT dependence in survivors, vasopressor use, organ dysfunction) compared to hemodialysis. Hemofiltration appeared to shorten time to filter failure (mean difference (MD) -7 hours, $95 \% \mathrm{Cl}(-19,+5), P=0.24$; two trials, $\mathrm{n}=50$ (primary analysis); $\mathrm{MD}-5$ hours, $95 \% \mathrm{Cl}(-10,-1), P=0.01$; three trials, $n=113$ (including combined hemofiltration-hemodialysis trials comparing similar doses); MD -6 hours, $95 \% \mathrm{Cl}(-10,-1), P=0.02$; five trials, $\mathrm{n}=383$ (sensitivity analysis)). Data primarily from crossover RCTs suggested that hemofiltration increased clearance of medium to larger molecules, including inflammatory cytokines, compared to hemodialysis, although almost no studies measured changes in serum concentrations. Meta-analyses were based on very limited data.

Conclusions: Data from small RCTs do not suggest beneficial clinical outcomes from hemofiltration, but confidence intervals were wide. Hemofiltration may increase clearance of medium to larger molecules. Larger trials are required to evaluate effects on clinical outcomes.
\end{abstract}

\section{Introduction}

Severe acute kidney injury (AKI) occurs in approximately $6 \%$ of patients admitted to an intensive care unit (ICU) [1] and in up to $19 \%$ of patients with vasopressordependent septic shock [2]. For such individuals, mortality is approximately $60 \%[1]$, and survivors are at

\footnotetext{
* Correspondence: j.friedrich@utoronto.ca

'Department of Medicine, University of Toronto, Toronto, ON, M5G 2C4, Canada

Full list of author information is available at the end of the article
}

increased risk of requiring permanent renal replacement therapy (RRT) [3]. Two multicenter randomized controlled trials (RCTs) [4,5] and two meta-analyses [6,7] have demonstrated that increasing the dose of RRT above 20 to $25 \mathrm{~mL} / \mathrm{kg} / \mathrm{h}$ of effluent flow for continuous renal replacement therapy (CRRT) or increasing intermittent dialysis frequency beyond alternate days does not improve survival.

In addition to dose, the mode of clearance is also a modifiable component of the RRT prescription that may

\section{Biomed Central}


affect patient outcomes. Convective clearance and diffusive clearance, delivered by hemofiltration and hemodialysis, respectively, can be provided by all continuous and some intermittent RRT machines. Despite similar clearance of small molecules, hemofiltration is reported to achieve higher clearance of medium-sized to larger molecules compared to hemodialysis [8]. Consequently, it is postulated that hemofiltration might benefit critically ill patients with AKI by better clearing large toxic inflammatory cytokines [9]. In the absence of a large, suitably-powered, randomized trial demonstrating the superiority of one mode over the other, practice surveys have shown variability in mode selection among countries and regions [10-15]. Therefore, our objective was to conduct a systematic review and meta-analysis of all RCTs comparing the effects of convective clearance (using hemofiltration) to diffusive clearance (using hemodialysis) in patients with AKI on clinically important outcomes.

\section{Materials and methods}

\section{Literature search}

We searched OVID versions of MEDLINE, EMBASE Classic and EMBASE and the Cochrane Central Register of Controlled Trials (from inception to June 2012) without language restrictions using a previously described search strategy [6]. We also searched abstracts from critical care and nephrology professional society conferences, including: Society of Critical Care Medicine (2004 to 2012), European Society of Intensive Care Medicine (2001 to 2011), International Symposium of Intensive Care and Emergency Medicine (2004 to 2012), American Thoracic Society (2004 to 2012), American College of Chest Physicians (2003 to 2011), American Society of Nephrology (2003 to 2011), and the European Renal Association - European Dialysis and Transplant Association (2002 to 2012). We also searched bibliographies of included studies and personal files. Two reviewers independently reviewed all citations and retrieved the full text of any citation considered potentially relevant by either reviewer. We attempted to contact selected authors of included studies for clarification of methods and to obtain additional data, where required.

\section{Study selection}

Two unblinded reviewers assessed full-text reports and included studies meeting the following criteria: (1) design: either parallel-group (patients assigned to only one treatment) or cross-over (each patient received both treatments in random order) randomized or quasi-randomized (for example, assigning patients in alternating fashion or by hospital registry number) controlled trial, (2) population: adult or post-neonatal pediatric patients with AKI requiring RRT, (3) intervention: hemofiltration compared to hemodialysis, with both modes applied using continuous or intermittent RRT; trials comparing continuous to intermittent RRT were excluded, (4) outcomes: all-cause mortality (primary outcome) or other clinically important outcomes (see below) for the parallel-group trials; or group-specific filter duration, or clearance or plasma concentration measurements of cleared substances for both parallel-group and crossover trials. We also included trials of hemodiafiltration (that is, combined dialysis-filtration) compared to either hemodialysis or hemofiltration in sensitivity analyses as explained below.

\section{Data abstraction and validity assessment}

Two unblinded reviewers independently abstracted data from included trials, including study population (number of centers, age, gender, illness acuity proportion with oliguria and sepsis, baseline creatinine and urea), RRT methods (modality, dose, duration of and criteria for starting/stopping study RRT), outcomes (mortality, RRT duration, RRT dependence in survivors, hemodynamics (for example, vasopressor doses), evolution of organ dysfunction, filter duration, measured clearances and plasma concentrations of metabolites) and study quality (including method of sequence generation and allocation concealment, intention-to-treat analysis, loss to follow-up for the outcome of mortality, and early trial stopping for efficacy before the planned enrollment was completed). For the crossover trials we also assessed whether carry-over effects and washout periods were addressed and whether paired data analyses were performed [16]. Disagreements between reviewers regarding study selection and data abstraction that remained after author contact were resolved by consensus.

\section{Data analysis}

Our primary outcome was all-cause hospital mortality and, if not available, then mortality at $90,60,30$ or 28 days after randomization, at ICU discharge or after stopping RRT (in descending order of preference). Secondary outcomes included RRT dependence among survivors at the latest time point available (with the same preferred order of time point as for the mortality analysis), RRT duration until renal recovery or death, filter duration, clearance of selected solutes and plasma concentration measurements.

Binary outcomes are reported as risk ratios (RR) and continuous outcomes using weighted mean differences (MD, a measure of absolute change) or ratio of means (RoM, a measure of relative change) [17]. We used Review Manager 5.1 (The Cochrane Collaboration, Oxford, England, UK) to calculate pooled outcome measures. We considered (two-sided) $P<0.05$ as statistically significant and reported individual trial and summary 
results with 95\% confidence intervals (CIs). We used random-effects models, which incorporate between-trial heterogeneity and give wider confidence intervals when heterogeneity is present, to pool data. We assessed statistical heterogeneity among trials using $I^{2}$, the percentage of total variability across studies due to heterogeneity rather than chance $[18,19]$, and used published thresholds to ascribe low $\left(I^{2}=25\right.$ to $\left.49 \%\right)$, moderate $\left(I^{2}=50\right.$ to $74 \%)$, and high $\left(I^{2} \geq 75 \%\right)$ heterogeneity [19]. Continuous variables are expressed as mean \pm standard deviation, unless otherwise indicated. Because the crossover trials generally did not report within-patient differences, we used the unpaired group-specific means to pool data, recognizing that this approach reduces the statistical power to detect differences [20].

For each outcome, the primary analysis included only trials in which one group was treated only with hemofiltration and the other group only with hemodialysis, with both groups receiving RRT providing similar (that is, within 20\%) small-molecule clearances. In assessing equivalency of doses, we determined whether trial authors adjusted for the reduced dose of hemofiltration that results from blood dilution by pre-filter replacement fluid, where applicable. In sensitivity analyses for each outcome, we included data from trials in which doses differed by greater than $20 \%$ between the convection and diffusion groups, and from trials comparing combined filtration-dialysis modes to either filtration or dialysis. For trials in which one group was treated with combined filtration-dialysis, this group was considered either as filtration (if compared to a dialysis-only group) or dialysis (if compared to a filtration-only group).

To assess for publication bias we planned to visually examine a funnel plot of study precision versus treatment effect on mortality for evidence of asymmetry, assuming $\geq 5$ trials in the analysis.

Because this study reports an analysis of published data, ethical approval was not required.

\section{Results}

\section{Study flow}

Our search strategy identified 6,657 citations, 6,324 from Medline, EMBASE Classic and EMBASE and 333 from the Cochrane Central Register of Controlled Trials. We retrieved 41 articles for detailed evaluation, of which 19 studies met criteria for inclusion [21-39] and 22 were excluded [40-61] (Figure 1). They included 10 parallelgroup trials [21-30] and 9 crossover trials [31-39]. Among the parallel-group trials, authors of 9 included trials [21,23-30] provided additional methodological or clinical data, and the author of the $10^{\text {th }}$ trial [22] informed us that no additional information was available. In addition, authors of two studies informed us either that the vast majority of enrolled randomized patients did not have renal failure [40], or that it was not a randomized controlled trial [41] (reference [62] is the full paper for the abstract reference [41]), leading to exclusion of these studies.

\section{Description of included studies (Table 1)}

Enrolled patients had high illness severity. The mean or median acute physiology and chronic health evaluation (APACHE) II score [63] was $\geq 20$ or simplified acute physiology score (SAPS) II [64] was $\geq 60$, as reported in 8 of 10 parallel-group trials [21-27,29] and 3 of 9 crossover trials [37-39]. One parallel-group trial reported only the mean sequential organ failure assessment (SOFA) [65] score of 16 [30]. Where reported, the vast majority of patients required mechanical ventilation or vasopressors [21,23,25,29-32,35,36]. AKI was defined by abnormal biochemistry (serum creatinine or urea) or a complication of oliguria (such as volume overload, hyperkalemia, acidosis or uremic symptoms) in 7 of 10 parallel-group trials $[21,23-26,29,30]$ and 3 of 9 crossover trials $[34,35,38]$. The remaining nine trials enrolled patients with AKI requiring RRT without reporting specific AKI criteria [22,27,28,31-33,36,37,39]. Five trials explicitly excluded patients with chronic kidney disease, defined by pre-morbid creatinine or creatinine clearance $[23,25,26,29]$ or recent dialysis [30],

\section{Description of RRT interventions (Table 2)}

Seven of the 10 parallel-group trials used continuous RRT [21,22,24-27,30-39], 1 trial used intermittent RRT [23] and 2 trials used sustained low efficiency RRT provided in sessions of eight hours each day $[28,29]$. Only three parallel-group trials, all using CRRT, $(\mathrm{n}=24$ [24], $\mathrm{n}$ $=20$ [25], $\mathrm{n}=78$ [30]) compared exclusively hemofiltration to exclusively hemodialysis at similar small-molecule doses. Of these, one trial allocated patients to higher ( 2.5 $\mathrm{L} / \mathrm{h})$ or lower $(1 \mathrm{~L} / \mathrm{h})$ dose continuous veno-venous hemofiltration $(\mathrm{CVVH})$ or continuous veno-venous hemodialysis $(C V V H D)$ in a $2 \times 2$ factorial design [24]. In six of the remaining seven parallel-group trials, patients treated with hemofiltration $(\mathrm{n}=20$ [21], $\mathrm{n}=206$ [26], $\mathrm{n}$ $=96$ [27]) or hemodialysis ( $\mathrm{n}=13$ [22], $\mathrm{n}=39$ [23], $\mathrm{n}=$ 60 [29]) were compared to a group treated with hemodiafiltration (hemofiltration and hemodialysis). Hemodiafiltration recipients received higher doses, except in three trials where doses in the two treatment arms were similar, although not corrected for lower clearance due to pre-filter addition of replacement fluid $[23,27,29]$. The seventh trial, which used sustained low efficiency RRT and was reported only in abstract form, randomized patients into three groups: two similar-dose groups of hemodialysis $(n=6)$ and hemodiafiltration $(n=5)$ and a lower-dose group of hemofiltration $(n=6)$ [28]. Only one parallel-group trial was multi-centered [30]. 

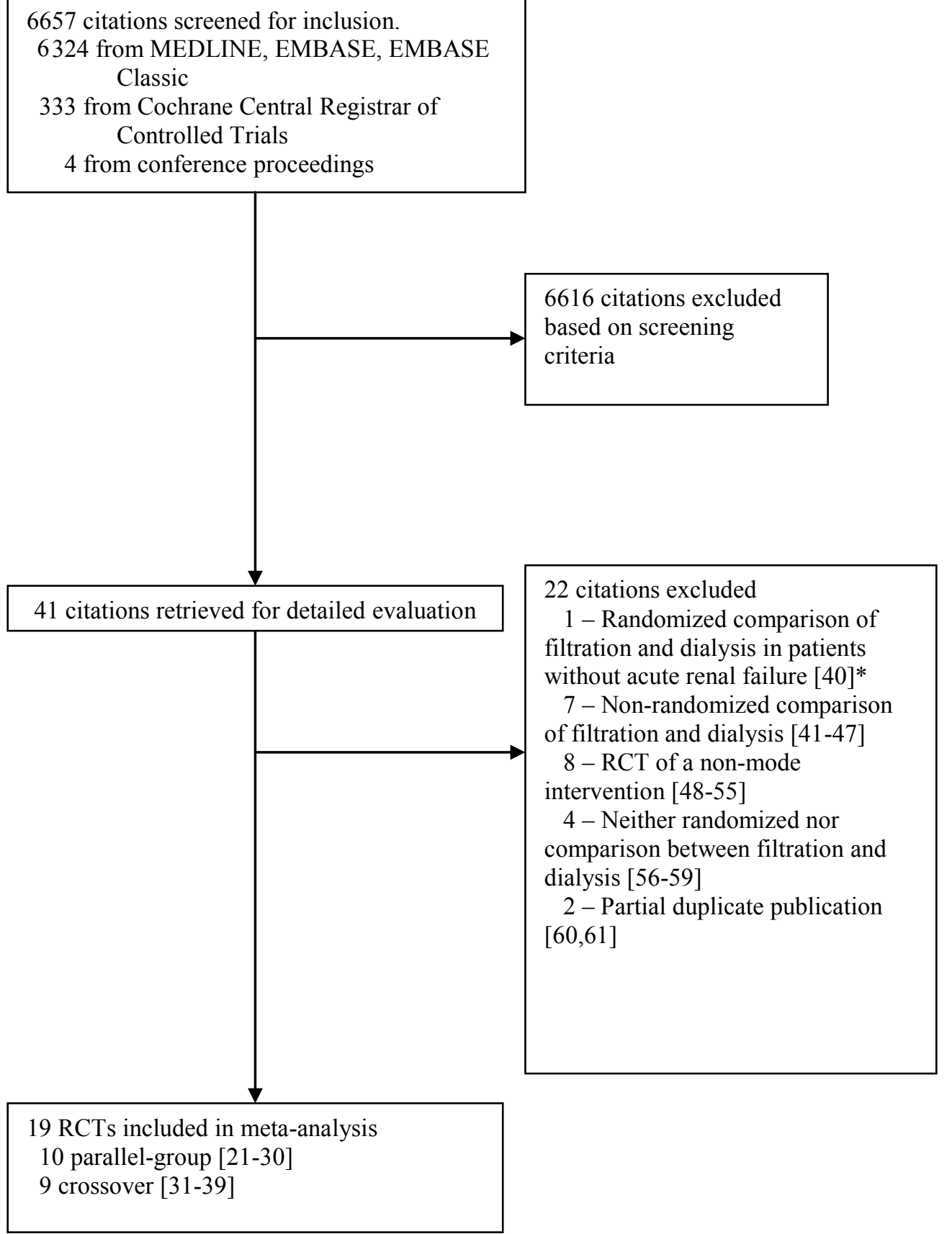

Figure 1 Flow chart of study selection. *In addition, author contact confirmed that mortality and other clinical outcomes not collected, and clearance rates not measured for this trial. Abbreviation: RCT, randomized controlled trial.

All crossover trials were single-centered, used CRRT, and included few randomized patients (median 11.5 patients (range, 5 to 31)) [31-39]. Six of nine crossover trials [31,33,34,36-38] compared hemofiltration and hemodialysis at similar doses. Of these, only three trials reported post-filter addition of replacement fluid [33,37] or appropriately adjusted the rate of pre-filter replacement fluid to compare similar clearances [38]. Patients in one of these three trials [37] received three treatments (post-filter hemofiltration, pre-filter hemofiltration and hemodialysis) in random order. In another trial [31], patients received hemofiltration and three doses of hemodialysis in random order. For this trial, we excluded the two higher dose hemodialysis groups and retained the two matched-dose hemofiltration and hemodialysis groups. 
Table 1 Patient characteristics in included trials

\begin{tabular}{|c|c|c|c|c|c|c|c|c|c|c|c|c|c|c|c|}
\hline \multirow[b]{2}{*}{ Trial } & \multirow[b]{2}{*}{$\begin{array}{l}\text { Patients, } \\
\text { n }\end{array}$} & \multirow[b]{2}{*}{$\begin{array}{c}\text { Centers, } \\
\mathrm{n}\end{array}$} & \multirow[b]{2}{*}{ Age,yr } & \multirow[b]{2}{*}{ Male\% } & \multirow[b]{2}{*}{$\begin{array}{c}\text { Surg } \\
\%\end{array}$} & \multicolumn{5}{|c|}{ Illness Acuity } & \multirow[b]{2}{*}{$\begin{array}{c}\text { Oliguria, } \\
\%\end{array}$} & \multirow[b]{2}{*}{$\mathrm{Cr}, \mu \mathrm{M}$} & \multirow[b]{2}{*}{$\begin{array}{l}\text { Urea, } \\
\mathrm{mM}\end{array}$} & \multirow[b]{2}{*}{$\begin{array}{l}\text { Sepsis, } \\
\%\end{array}$} & \multirow[b]{2}{*}{$\begin{array}{c}\text { Mortality }{ }^{a} \text {, } \\
\%\end{array}$} \\
\hline & & & & & & $\begin{array}{l}\text { APACHE } \\
\text { II }\end{array}$ & $\begin{array}{c}\text { SAPS } \\
\text { II }\end{array}$ & $\begin{array}{l}\text { MODS or } \\
\text { SOFA }\end{array}$ & $\begin{array}{c}\text { Ventilated } \\
\%\end{array}$ & $\begin{array}{c}\text { Vasopressors } \\
\%\end{array}$ & & & & & \\
\hline \multicolumn{16}{|l|}{ Parallel Group } \\
\hline $\begin{array}{l}\text { Davenport } 1993 \text { [21] } \\
\text { (medians) }\end{array}$ & 20 & 1 & 33 & $45 \%$ & & 28 & & & $100 \%$ & & $100 \%^{b}$ & 646 & 18 & & $84 \%^{h}$ \\
\hline Alamartine 1994 [22] & 13 & 1 & $n / r$ & $n / r$ & & 22 & & & & & & 566 & 30 & & $\mathrm{n} / \mathrm{a}$ \\
\hline Pettila 2001 [23] & 39 & 1 & 48 & $82 \%$ & & 20 & & 10 MODS & $95 \%$ & & $61 \%^{\mathrm{c}}$ & 446 & 31 & & $42 \%$ \\
\hline $\begin{array}{l}\text { Morgera } 2004 \text { [24] } \\
\text { (medians) }\end{array}$ & 24 & 1 & 65 & $58 \%$ & & 31 & & & & & & 254 & 44 & & $50 \%(I C U)$ \\
\hline Daud 2006 [25] (medians) & 20 & 2 & 50 & $60 \%$ & & & 66 & & $100 \%$ & & & 265 & 19 & $90 \%$ & $85 \%(I C U)$ \\
\hline Saudan 2006 [26] & 206 & 1 & 63 & $61 \%$ & & 25 & & 9 SOFA & & & $37 \%^{d}$ & 428 & 30 & $60 \%$ & $53 \%(90 d)$ \\
\hline Chang 2009' [27] & 96 & 1 & 65 & $57 \%$ & & 31 & & 13 SOFA & & & & 238 & 19 & $100 \%$ & $54 \%(28 d)$ \\
\hline Ratanarat 2009 [28] & 17 & 1 & 65 & & & & & & & & & & & & $n / c$ \\
\hline Ratanarat 2012 [29] & 60 & 1 & 57 & $60 \%$ & $0 \%$ & 27 & & & $93 \%$ & $67 \%$ & & 449 & 32 & $82 \%$ & $47 \%(28 d)$ \\
\hline OMAKI 2012 [30] & 77 & 6 & 61 & $61 \%$ & & & & 16 SOFA & $95 \%$ & $90 \%$ & & 261 & 22 & $82 \%$ & $55 \%$ \\
\hline \multicolumn{16}{|l|}{ Crossover } \\
\hline Maher 1988 [31] & 5 & 1 & $\begin{array}{c}\text { median } \\
51\end{array}$ & $100 \%$ & & & & & $80 \%$ & $100 \%$ & & & & & \\
\hline Alarabi 1992 [32] & 13 & 1 & 60 & $62 \%$ & $38 \%$ & & & & $92 \%$ & $100 \%$ & & 453 & 36 & & \\
\hline Jeffery 1994 [33] & 10 & 1 & & & & & & & & & & & & & \\
\hline Kellum 1998 [34] & $13(10)^{e}$ & 1 & & & & & & & & & & & & $100 \%$ & $77(70) \%^{e}$ \\
\hline Garcia-Fernandez 2000 [35] & 40 & 1 & 60 & $65 \%$ & $38 \%$ & & & & $58 \%$ & $53 \%$ & $70 \%^{i}$ & & & $48 \%$ & $48 \%$ \\
\hline Maxvold 2000 [36] & 6 & 1 & 11 & $33 \%$ & $17 \%$ & & & & & $100 \%$ & & & & & $\begin{array}{l}50 \%( \\
\text { undef) }\end{array}$ \\
\hline Wynkel 2004 [37] & 18 & 1 & 62 & & $39 \%$ & & $60^{9}$ & & & & & & & & $50 \%(30 d)$ \\
\hline Ricci 2006 [38] (medians) & 15 & 1 & 50 & $67 \%$ & $60 \%$ & & 61 & & & & & & & & \\
\hline Davies 2008 [39] & $45(31)^{f}$ & 1 & $61(57)$ & $\begin{array}{l}64 \% \\
(77 \%)\end{array}$ & $38 \%$ & 26 & & & & & & & & & \\
\hline
\end{tabular}

Means, (medians where noted). ${ }^{a}$ Hospital mortality except where noted. Oliguria: ${ }^{\mathrm{b}}<10 \mathrm{~mL} / \mathrm{h}$ [21], ${ }^{\mathrm{c}}<500 \mathrm{~mL} / 24 \mathrm{~h}$ [23], ${ }^{\mathrm{d}}<200 \mathrm{~mL} / 12 \mathrm{~h}$ [26], and ${ }^{\mathrm{i}}<400 \mathrm{~mL} / 24 \mathrm{~h}$ [34]. ${ }^{\mathrm{e}}$ For Kellum 1998 [34], 3/13 patients died prior to being crossed over to other arm. ${ }^{\mathrm{F} F o r}$ Davies 2008 [39], 45 patients were randomized but only 31 patients crossed over and received both therapies. ${ }^{9}$ Wynkel 2004 [37] excluded SAPS II >= 85. ${ }^{h}$ Mortality obtained after author contact [21]. ${ }^{\text {j}}$ Chang 2009 [27] reported data from 65 patients in abstract form; data for 31 patients who were subsequently randomized provided after author contact. ${ }^{k}$ Author informed us that mortality was not recorded [28]. Abbreviations: APACHE II, acute physiology and chronic health evaluation II [63]; Cr, serum creatinine concentration in micromoles per litre; MODS, multiple organ dysfunction score [66]; $n / a$, not available after author contact; $\mathrm{n} / \mathrm{c}$, not collected; $\mathrm{n} / \mathrm{r}$, not reported; SAPS II, simplified acute physiology score II [64]; SOFA, sequential organ failure assessment score [65]. 
Table 2 Renal replacement intervention in the included trials

\begin{tabular}{|c|c|c|c|c|c|c|c|c|c|c|}
\hline \multirow[b]{2}{*}{ Trial } & \multirow[b]{2}{*}{ Cessation of study RRT } & \multirow[b]{2}{*}{ Filter } & \multirow[b]{2}{*}{$\begin{array}{c}\text { Anti- } \\
\text { coagulation }\end{array}$} & \multirow[b]{2}{*}{$\begin{array}{l}\text { Blood } \\
\text { flow,mL/ } \\
\text { min }\end{array}$} & \multicolumn{2}{|c|}{ Filtration only group } & \multicolumn{2}{|c|}{$\begin{array}{l}\text { Combined filtration and } \\
\text { dialysis group }\end{array}$} & \multicolumn{2}{|c|}{ Dialysis only group } \\
\hline & & & & & Mode $^{b}$ & $\begin{array}{l}\text { Dose, }^{c} \text { L/h; Study } \\
\text { RRT duration }\end{array}$ & Mode $^{\mathrm{b}}$ & $\begin{array}{l}\text { Dose, }^{c} \mathrm{~L} / \mathrm{h} \\
\text { (or d); } \\
\text { Study RRT } \\
\text { duration }^{\mathrm{j}}\end{array}$ & Mode & $\begin{array}{l}\text { Dose, }{ }^{c} \text { L/h (or } \\
\text { d); Study RRT } \\
\text { duration }\end{array}$ \\
\hline \multicolumn{11}{|l|}{$\begin{array}{l}\text { Parallel } \\
\text { Group }\end{array}$} \\
\hline $\begin{array}{l}\text { Davenport } \\
1993[21]\end{array}$ & Clinician & FH77 (Gambro) & UFH & $n / r$ & CAVH (post) & 0.9 L/h (median) & $\begin{array}{l}\text { CAVHDF } \\
\text { (post) }\end{array}$ & $\begin{array}{l}1 \text { (D) }+0.4 \\
\text { (F) } L / h \\
\text { (median) }\end{array}$ & & \\
\hline $\begin{array}{l}\text { Alamartine } \\
1994[22]\end{array}$ & Clinician & $\begin{array}{l}0.90 \mathrm{~m}^{2} \text { F50 poly-sulfone } \\
\text { (Fresenius-Smad) }\end{array}$ & UFH & 150 & & & $\begin{array}{l}\text { CWHDF } \\
\text { (pre) }\end{array}$ & $\begin{array}{l}1(\mathrm{D})+2(\mathrm{~F}) \\
\mathrm{L} / \mathrm{h}\end{array}$ & CWHD & $1 \mathrm{~L} / \mathrm{h}$ \\
\hline $\begin{array}{l}\text { Pettila } \\
2001 \text { [23] }\end{array}$ & Clinician & Polyflux 17 & LMWH & 250 & & & IHDF (pre) & $\begin{array}{l}40(F)+128 \\
\text { (D) L/d; } 23.5 \\
\pm 18.8 d\end{array}$ & $\mathrm{IHD}$ & $\begin{array}{l}164(D)+4(F) L / \\
d ; 17.5 \pm 9.2 d\end{array}$ \\
\hline $\begin{array}{l}\text { Morgera } \\
2004[24]^{d}\end{array}$ & Maximum of 3 days & $\begin{array}{l}\text { Polyflux P2SH } 1.1 \mathrm{~m}^{2}, 10 \mathrm{~nm} \\
\text { pore, } 50 \mu \mathrm{m} \text { membrane } \\
\text { thickness; } 60 \mathrm{kDa} \text { cutoff }\end{array}$ & UFH & $n / r$ & CWH (post) & 1 or $2.5 \mathrm{~L} / \mathrm{h} ; 2.8 \mathrm{~d}$ & & & CWHD & 1 or $2.5 \mathrm{~L} / \mathrm{h} ; 2.8 \mathrm{~d}$ \\
\hline $\begin{array}{l}\text { Daud } 2006 \\
\text { [25] }\end{array}$ & Clinician & AN69 $0.6 \mathrm{~m}^{2}$, changed daily & UFH & $\geq 120$ & CWH (pre) & $\begin{array}{c}2 \mathrm{~L} / \mathrm{h} ; 2.0 \text { (IQR 5.1) } \\
\mathrm{d}\end{array}$ & & & CWHD & $\begin{array}{c}1.7 \mathrm{~L} / \mathrm{h} ; 1.9(\mathrm{IQR} \\
2.2) \mathrm{d}\end{array}$ \\
\hline $\begin{array}{l}\text { Saudan } \\
2006[26]\end{array}$ & Protocol & $\begin{array}{l}\text { AN69 } 0.9 \mathrm{~m}^{2} \text { (Gambro) } \\
\text { changed daily } \times 2 \mathrm{~d}\end{array}$ & UFH & $\begin{array}{l}100 \text { to } \\
125\end{array}$ & CWH (pre) & $\begin{array}{c}1.8 \mathrm{~L} / \mathrm{h}(25 \mathrm{~mL} / \mathrm{kg} / \\
\mathrm{h})\end{array}$ & $\begin{array}{l}\text { CWHDF } \\
\text { (pre) }\end{array}$ & $\begin{array}{l}3.1 \mathrm{~L} / \mathrm{h}(24 \\
(\mathrm{F})+18(\mathrm{D}) \\
\mathrm{mL} / \mathrm{kg} / \mathrm{h})\end{array}$ & & \\
\hline $\begin{array}{l}\text { Chang } \\
2009[27]\end{array}$ & Clinician & multiflow 100 (Gambro) & $\begin{array}{l}\text { UFH or no } \\
\text { anticoag if } \\
\text { high risk } \\
\text { bleed }\end{array}$ & $\begin{array}{l}100 \text { to } \\
150\end{array}$ & CWH (pre) & $\begin{array}{c}2.4^{\mathrm{k}} \mathrm{L} / \mathrm{h}(40 \mathrm{~mL} / \mathrm{kg} / \\
\mathrm{h}) ; 7.4 \pm 8.0 \mathrm{~d}\end{array}$ & $\begin{array}{l}\text { CWHDF } \\
\text { (pre) }\end{array}$ & $\begin{array}{c}2.4^{\mathrm{k}} \mathrm{L} / \mathrm{h}(20 \\
{[\mathrm{F}]+20[\mathrm{D}]} \\
\mathrm{mL} / \mathrm{kg} / \mathrm{h}) \\
8.5 \pm 8.9 \mathrm{~d}\end{array}$ & & \\
\hline $\begin{array}{l}\text { Ratanarat } \\
2009[28]^{1}\end{array}$ & Maximum 3 days & F40s or F80s' & $n / r$ & $\begin{array}{l}200 \text { to } \\
250 \text { or } \\
250 \text { to } \\
300^{\prime}\end{array}$ & $\begin{array}{l}\text { Sustained } \\
\text { low efficency } \\
\text { filtration (pre) }\end{array}$ & $\begin{array}{c}39 \pm 6^{\prime} \mathrm{L} / \mathrm{d}(1.25 \times \\
\text { body weight (in kg) } \\
\mathrm{mL} / \mathrm{min})\end{array}$ & $\begin{array}{l}\text { Sustained } \\
\text { low } \\
\text { efficiency } \\
\text { diafiltration } \\
\text { (pre) }\end{array}$ & $\begin{array}{l}48(F)+96 \\
\text { (D) } L / d\end{array}$ & $\begin{array}{l}\text { sustained } \\
\text { low } \\
\text { efficiency } \\
\text { dialysis }\end{array}$ & $144 \mathrm{~L} / \mathrm{d}$ \\
\hline $\begin{array}{l}\text { Ratanarat } \\
2012[29]\end{array}$ & Clinician & $\begin{array}{l}\text { HF80S }\left(1.8 \mathrm{~m}^{2}, \text { Kuf } 55 \mathrm{~mL} / \mathrm{h}\right. \\
\times \mathrm{mm} \mathrm{Hg}) \text { (Fresenius) }\end{array}$ & UFH & 250 & & & $\begin{array}{l}\text { Sustained } \\
\text { low } \\
\text { efficiency } \\
\text { diafiltration } \\
\text { (pre) }\end{array}$ & $\begin{array}{l}48(F)+96 \\
\text { (D) L/d }\end{array}$ & $\begin{array}{l}\text { Sustained } \\
\text { low } \\
\text { efficiency } \\
\text { dialysis }\end{array}$ & $144 \mathrm{~L} / \mathrm{d}$ \\
\hline $\begin{array}{l}\text { OMAKI } \\
2012[30]\end{array}$ & $\begin{array}{l}\mathrm{U} / \mathrm{O}>500 \mathrm{~mL} / 12 \mathrm{~h}, \mathrm{~K}<5.5 \mathrm{mM} \\
\text { and } \mathrm{HCO}_{3}>18 \mathrm{mM} \text {; or } \\
\text { stepdown to IHD when SOFA } \\
\text { CV score }<2 \text { for }>24 \mathrm{~h} ;\end{array}$ & $\begin{array}{l}\text { AN69 ST100 }\left(1 \mathrm{~m}^{2}\right) \text { or ST150 } \\
\left(1.5 \mathrm{~m}^{2}\right) \text { (Gambro) }\end{array}$ & $\begin{array}{l}\text { UFH or } \\
\text { citrate or no } \\
\text { anticoag. }\end{array}$ & $\geq 150$ & $\begin{array}{l}\text { CWH } \\
\text { (equally } \\
\text { distributed } \\
\text { pre/post) }\end{array}$ & $\begin{array}{l}\text { 3.0.L/h (2.7 L/h post } \\
\text { equiv.) }(34 \mathrm{~mL} / \mathrm{kg} / \\
\text { h); } 5 \text { (IQR } 3 \text { to } 7) \mathrm{d}\end{array}$ & & & CWHD & $\begin{array}{l}3.1 \mathrm{~L} / \mathrm{h} \text { (incl. } 0.2 \\
\mathrm{~L} / \mathrm{h} \mathrm{F} \text { post) }(35 \\
\mathrm{mL} / \mathrm{kg} / \mathrm{h}) ; 4.5(\mathrm{IQR} \\
3 \text { to } 10.25) \mathrm{d}\end{array}$ \\
\hline \multicolumn{11}{|l|}{ Crossover } \\
\hline $\begin{array}{l}\text { Maher } \\
1988[31]^{e}\end{array}$ & $n / r$ & $\begin{array}{c}\text { FH55 HF or AN69S HD } \\
\text { (Gambro) }\end{array}$ & $n / r$ & $n / r$ & CAVH $(n / r)$ & $1 \mathrm{~L} / \mathrm{h}$ & & & CAVHD & $1 \mathrm{~L} / \mathrm{h}$ \\
\hline
\end{tabular}


Table 2 Renal replacement intervention in the included trials (Continued)

\begin{tabular}{|c|c|c|c|c|c|c|c|c|c|c|}
\hline $\begin{array}{l}\text { Alarabi } \\
1992[32]^{f}\end{array}$ & $24 \mathrm{~h}$ & $\begin{array}{l}\text { Polysulphone (Amicon } \\
\text { AMD30) }\end{array}$ & UFH & $n / r$ & CAVH (post) & 0.833L/h (median) & $\begin{array}{l}\text { CAVHDF } \\
\text { (post) }\end{array}$ & $\begin{array}{c}0.9 \mathrm{D}+ \\
0.313 \mathrm{~F} \mathrm{L/h} \\
\text { (median) }\end{array}$ & & \\
\hline $\begin{array}{l}\text { Jeffery } \\
1994 \text { [33] }\end{array}$ & $0.5 \mathrm{~h}$ & AN69 Filtral 10 & $n / r$ & 200 & $\mathrm{CWH}$ (post) & $1.5 \mathrm{~L} / \mathrm{h}$ & & & CWHD & $1.5 \mathrm{~L} / \mathrm{h}$ \\
\hline $\begin{array}{l}\text { Kellum } \\
1998[34]^{9}\end{array}$ & $24 \mathrm{~h}$ & AN69 $\left(0.6 \mathrm{~m}^{2}\right)$ & UFH & $\begin{array}{l}150 \text { to } \\
200\end{array}$ & $\mathrm{CWH}(\mathrm{n} / \mathrm{r})$ & $2 \mathrm{~L} / \mathrm{h}$ & & & CWHD & $2 \mathrm{~L} / \mathrm{h}$ \\
\hline $\begin{array}{l}\text { Garcia- } \\
\text { Fernandez } \\
2000 \text { [35] }\end{array}$ & $24 \mathrm{~h}$ & $\begin{array}{l}\text { High Flux polysulfone (0.6 } \\
m^{2} \text { ) Bellco, Sorin Biomedica }\end{array}$ & UFH & $\begin{array}{l}100 \text { to } \\
150\end{array}$ & CWH (post) & $0.71 \mathrm{~L} / \mathrm{h}$ (median) & $\begin{array}{l}\text { CWHDF } \\
\text { (post) }\end{array}$ & $\begin{array}{l}1 \mathrm{D}+0.56 \mathrm{~F} \\
\mathrm{~L} / \mathrm{h} \text { (median) }\end{array}$ & & \\
\hline $\begin{array}{l}\text { Maxvold } \\
2000[36]\end{array}$ & $24 \mathrm{~h}$ & $\begin{array}{l}\text { Polysulfone hemofilt. HF-400, } \\
\text { Renal Systems }\end{array}$ & UFH & $\begin{array}{c}4(\mathrm{~mL} / \\
\mathrm{kg} / \mathrm{min})\end{array}$ & CWH (pre) & $2^{h} L / h$ & & & CWHD & $2^{h} L / h$ \\
\hline $\begin{array}{l}\text { Wynkel } \\
2004[37]\end{array}$ & $24 \mathrm{~h}$ & $\begin{array}{l}\text { AN69S, M100 }\left(0.9 \mathrm{~m}^{2}\right) \\
\text { changed daily }\end{array}$ & $\begin{array}{l}\text { UFH or } \\
\text { LMWH }\end{array}$ & 150 & $\begin{array}{l}\mathrm{CWH}(\text { pre } \\
\text { and post) }\end{array}$ & $1.5 \mathrm{~L} / \mathrm{h}$ & & & CWHD & $1.5 \mathrm{~L} / \mathrm{h}$ \\
\hline $\begin{array}{l}\text { Ricci } 2006 \\
{[38]}\end{array}$ & Filter failure & $\begin{array}{c}\text { AN69 }\left(0.9 \mathrm{~m}^{2}\right) \text { multiflow } 100 \text {, } \\
\text { Hospel, UF coefficient with } \\
\text { blood } 25 \mathrm{~mL} / \mathrm{h} / \mathrm{mm} \mathrm{Hg} \times \\
\mathrm{m}^{2} ; 40 \mathrm{kDa} \text { cutoff }\end{array}$ & UFH & $\begin{array}{l}150(F) \\
\text { and } 135 \\
\text { (D) } \\
\text { (medians) }\end{array}$ & $\begin{array}{l}\text { CWH (pre/ } \\
\text { post to keep } \\
\text { filtration } \\
\text { fraction } \\
<20 \%)\end{array}$ & $\begin{array}{l}35 \mathrm{~mL} / \mathrm{kg} / \mathrm{h}(1.45 \mathrm{~L} / \\
\mathrm{h} \text { pre }+1.5 \mathrm{~L} / \mathrm{h} \\
\text { post (medians)); } 19 \\
(\text { IQR 12.5, 28) h }\end{array}$ & & & CWHD & $\begin{array}{l}35 \mathrm{~mL} / \mathrm{kg} / \mathrm{h}(2.15 \\
\text { L/h (median)); } 37 \\
\text { (IQR 19.5, 72.5) h }\end{array}$ \\
\hline $\begin{array}{l}\text { Davies } \\
2008 \text { [39] }\end{array}$ & Filter failure & $\begin{array}{c}\text { AN69 (Nephral 300ST, } \\
\text { Hospal) }\end{array}$ & UFH & $\begin{array}{l}150 \text { to } \\
200\end{array}$ & CWH (pre) & $\begin{array}{l}35 \mathrm{~mL} / \mathrm{kg} / \mathrm{h} \text { (mean } \\
3.1 \mathrm{~L} / \mathrm{h} \text { ); } 8.6 \pm 5.6 \mathrm{~h}\end{array}$ & $\begin{array}{l}\text { CWHDF } \\
\text { (pre) }\end{array}$ & $\begin{array}{c}1 \mathrm{D}+0.6 \mathrm{~F} \\
\mathrm{~L} / \mathrm{h} ; 18.7 \pm \\
3.1 \mathrm{~h}\end{array}$ & & \\
\hline
\end{tabular}

${ }^{a}$ Cessation refers to discontinuation of study renal replacement therapy by clinician discretion, or when a fixed time point was reached. For the crossover trials the fixed time point refers to the time prior and after the crossover (that is, duration of each mode). " $F$ For filtration modes, "pre" and "post" refer to pre- and post-filter infusion of replacement fluid if reported. "For trials of continuous renal replacement therapy, effluent flow rates are as reported in the publications assuming no net fluid removal and without adjustment for reduced clearance due to prefilter replacement fluid (for CAVH, CVVH, CAVHDF, or CVVHDF, or IDHF) except where noted [30]. ${ }^{\mathrm{D} F o r}$ Morgera 2004 [24], patients were randomized to 1 of 4 groups: 1 or $2.5 \mathrm{~L} / \mathrm{h}$ of CVVH or 1 or $2.5 \mathrm{~L} / \mathrm{h}$ of CVVHD. ${ }^{\mathrm{e}}$ For Maher 1988 [31], patients were also randomly crossed over to 2 additional CAVHD dose groups: 1.5 and 2 L/h of CAVHD. 'For Alarabi 1992 [32], patients were crossed over twice and received three treatments each over 24 hours, either CAVH-CAVHDF-CAVH or CAVHDF-CAVH-CAVHDF. We only included data from the first two treatments for each patient. ${ }^{9}$ For Kellum 1998 [34], we only included data from the 10/13 patients that received both treatments (3/13 patients died prior to being crossed over). $h^{h}$ For the pediatric patients in Maxvold 2000 [36], dose is expressed in L/h/1.73 $\mathrm{m}^{2}$ body surface area. 'For Wynkel 2004 [37], patients were randomly assigned to receive three treatments each over 24 hours in one of the following orders: CVVHD/CVVHpre/CVVHpost, CVVHpre/CVVHpost/CVVHD, or CVVHpost/CVVHD/CVVHpre. ${ }^{j}$ For parallel group trials; and for crossover trials that specified duration of RRT based on filter failure and not a fixed duration of time. ${ }^{~}$ Calculated using mean weight of $59 \mathrm{~kg}$ obtained after author contact [27]. 'Data provided after author contact [28]. Randomized into three groups each treated for 8 hours per day: dialysis only ("sustained low efficiency daily dialysis" group in published abstract), diafiltration ("sustained low efficiency daily diafiltration" group), and filtration only ("predilution hemofiltration" group). F40s filter used for the dialysisonly group, and F80s for the diafiltration and filtration-only groups. Blood flow of 200 to $250 \mathrm{~mL} / \mathrm{min}$ in the dialysis - only and difiltration groups, and 250 to $300 \mathrm{~mL} / \mathrm{min}$ in the filtration-only group. Filtration dose in the filtration-only group (in $\mathrm{mL} / \mathrm{min}$ ) was set at $1.25 \times$ body weight (in $\mathrm{kg}$ ) and body weight in this group was $64.4 \pm 10.2$ (mean \pm standard deviation).

Abbreviations: CAVH, continuous arteriovenous hemofiltration; CAVHD, continuous arteriovenous hemodialysis; CAVHDF, continuous arteriovenous hemodiafiltration; CV, cardiovascular component of SOFA [65] score; CVVH, continuous venovenous hemofiltration; CVVHD, continuous venovenous hemodialysis; CVVHDF, continuous venovenous hemodiafiltration; $d$, days; equiv., equivalent; $D$, dialysis dose; $F$, filtration dose; $h$, hours; $\mathrm{HCO}_{3}$, serum bicarbonate concentration; IHD, intermittent hemodialysis; IHDF, intermittent hemodiafiltration; IQR, interquartile range; $\mathrm{K}$, serum potassium concentration; $\mathrm{kDa}$, kiloDalton; $\mathrm{kg}$, body weight in kilograms;

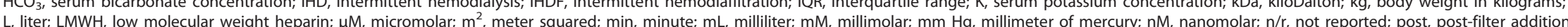
of replacement fluid in hemofiltration modes; pre, pre-filter addition of replacement fluid in hemofiltration modes; RRT, renal replacement therapy; SOFA, mean sequential organ failure assessment score [65]; U/O, urine output; UFH, unfractionated heparin 
In the remaining three crossover trials, hemofiltration was compared to a mixture of hemofiltration and hemodialysis at either higher [32,35] or lower [39] doses. In one of these trials [32], patients were crossed over twice and received their initial treatment a second time; only data from the first two treatments were included.

One parallel-group trial used different fixed doses based on weight ranges [26] while two others used weight-based dosing $[27,30]$. The parallel-group trials with sustained low efficiency RRT used fixed doses for the hemodialysis and hemodiafiltration groups [29] and weight-based dosing for the hemofiltration group [28]. One crossover trial used weight-based dose prescriptions for both interventions [38], while another used it only for hemofiltration [39]. The remaining trials used either fixed doses [22-25,31,33,34,36,37] or dose ranges not directly related to patient weight $[21,32,35]$.

Among the parallel-group trials, RRT was discontinued at the clinicians' discretion [21-23,25,27,29], after a fixed duration $[24,28]$, or when protocol-defined criteria were met $[26,30]$. The mean duration of RRT ranged from 2 to 8 days in four parallel-group CRRT trials $[24,25,27,30]$ and just over 20 days in the parallel-group with intermittent therapy [23]. Among the crossover trials, patients were crossed over after a fixed time: $24 \mathrm{~h}$ for most trials [32,34-37] and $0.5 \mathrm{~h}$ for one trial [33]. Two trials crossed patients over after filter clotting or failure $[38,39]$. One trial did not report when patients were crossed over [31].

\section{Study quality (Table 3)}

In the parallel-group trials that provided these data, all patients were analyzed according to the group to which they were initially assigned, and withdrawal of randomized patients from the mortality analysis either did not occur $[26,27]$ or comprised $\leq 5 \%$ of randomized patients $[21,23,30]$. Caregiver blinding was not practical in any trial, given the nature of the intervention. Four trials reported $[21,23,26,30]$ concealed allocation. In three trials reporting mortality $[24,25,27]$ the authors informed us that they allocated patients to interventions in an alternating manner. The author of one trial informed us that the trial stopped early for benefit [26]. This trial was included only in the sensitivity analyses due to the use of differing doses and a mixture of hemofiltration and hemodialysis. Quality measures shown in Table 3 were generally not reported for the crossover trials, although in two crossover trials, authors reported that $3 / 13(23 \%)$ [34] and 14/45 (31\%) [39] patients were not crossed over to the other treatment. Carryover effects were generally ignored, which may have been reasonable given that the impact of clearance mode on solute removal should manifest relatively quickly [8], and subsequent clearance and concentration measurements were collected over a period of 12 to 24 hours after crossover in most trials. The trial with the shortest measurement period (30 minutes) provided a 10-minute equilibration period after crossover prior to data collection [33]. For one other trial [37], we included data collected over 12 hours but not starting until 12 hours after crossover. Most crossover trials reported using paired analyses [33-37,39], but none provided individual patient data or mean within-patient difference data. Therefore, we were restricted to using group-specific means to perform meta-analyses, as discussed in the Methods.

\section{Clinical outcomes}

The three parallel group trials that compared similar doses of hemofiltration to hemodialysis were included in the primary analysis and reported either ICU $[24,25]$ or hospital [30] mortality. Five additional parallel-group trials comparing similar $[23,27,29]$ or different $[21,26]$ doses of hemodiafiltration to either hemodialysis or hemofiltration reported mortality data and were included in the sensitivity analysis. They reported hospital [21,23], 28-day [27,29], or 90-day [26] mortality. We did not identify differences in pooled mortality in the primary (RR $0.96,95 \%$ CI 0.73 to $1.25, P=0.76$; three trials, 121 patients) or sensitivity (RR $1.10,95 \%$ CI 0.88 to $1.38, P=0.38$; eight trials, 540 patients) analyses (Figure 2). Statistical heterogeneity was absent $\left(I^{2}=0 \%\right)$ and moderate $\left(I^{2}=50 \%\right)$, respectively. Visual inspection of the funnel plot for the sensitivity analysis did not suggest publication bias.

Dialysis dependence in survivors was not different between groups (primary analysis: RR 1.33, 95\% CI 0.35 to $5.08, P=0.67$; two trials $[25,30], 37$ surviving patients; sensitivity analysis: RR $0.95,95 \%$ CI 0.44 to $2.04, P=0.89$; four trials [25-27,30], 177 surviving patients), with no statistical heterogeneity $\left(I^{2}=0 \%\right)$. Two trials reported no differences in SOFA scores 72 hours after starting therapy (primary analysis: RoM 1.02, 95\% CI 0.89 to $1.16, P=0.79$; one trial, 63 patients [30]; sensitivity analysis: RoM $1.00,95 \%$ CI 0.61 to $1.64, P=$ 0.99; two trials, 124 patients $[27,30])$. One trial reported lower SOFA scores in the hemofiltration group primarily between four and seven days, driven primarily by the cardiovascular component (reflecting lower vasopressor requirements); however, this decrease was not statistically significant [30]. Another trial reported no difference in norepinephrine doses or changes in the multiorgan dysfunction score (MODS) [66] or APACHE II score between continuous hemofiltration and hemodialysis groups over the first 72 hours [24]. One parallelgroup trial, comparing intermittent hemodiafiltration to hemodialysis, reported similar improvements in MODS between groups over 10 days [23], and another comparing sustained low efficiency dialysis to diafiltration reported similar improvements in blood pressure between groups over three days [29]. 
Table 3 Risk of bias of included trials

\begin{tabular}{|c|c|c|c|c|c|}
\hline Trial & $\begin{array}{l}\text { Sequence } \\
\text { generation }\end{array}$ & Concealment of allocation & $\begin{array}{l}\text { Trial stopped early } \\
\text { for benefit }\end{array}$ & $\begin{array}{l}\text { Intention to treat } \\
\text { analysis }\end{array}$ & $\begin{array}{l}\text { Post-randomization withdrawals for mortality analysis (parallel } \\
\text { group trials) or not crossed over }\end{array}$ \\
\hline \multicolumn{6}{|l|}{ Parallel group } \\
\hline $\begin{array}{l}\text { Davenport } 1993 \\
\text { [21] }\end{array}$ & $\begin{array}{l}\text { List of random } \\
\text { numbers }\end{array}$ & Yes $^{a}$ & No & Yes & Yes - 1/12 CWHD(lost to follow up) \\
\hline $\begin{array}{l}\text { Alamartine } 1994 \\
{[22]^{\mathrm{a}}}\end{array}$ & $n / r$ & $n / r$ & $n / r$ & $n / r$ & $n / r$ \\
\hline Pettila 2001 [23] & $\begin{array}{l}\text { Computer } \\
\text { generated }\end{array}$ & Yes $^{\mathrm{a}}$ (closed envelopes) & No & Yes & Yes - 1/18 IHD(consent withdrawn) \\
\hline Morgera 2004 [24] & $\begin{array}{l}\text { Alternating } \\
\text { patients }^{\mathrm{a}}\end{array}$ & No & No & Yes & $n / r$ \\
\hline Daud 2006 [25] & $\begin{array}{l}\text { Alternating } \\
\text { patients }^{\mathrm{a}}\end{array}$ & No & No & Yes & $n / r$ \\
\hline Saudan 2006 [26] & $\begin{array}{l}\text { Computer } \\
\text { generated }\end{array}$ & $\begin{array}{l}\text { Yes (sequentially numbered sealed } \\
\text { opaque envelopes) }\end{array}$ & Yes $^{a}$ & Yes & No \\
\hline Chang 2009 [27] & $\begin{array}{l}\text { Alternating } \\
\text { patients }^{\mathrm{a}}\end{array}$ & No & $\mathrm{No}^{\mathrm{a}}$ & Yes & $\mathrm{No}^{\mathrm{a}}$ \\
\hline $\begin{array}{l}\text { Ratanarat } 2009 \\
{[28]}\end{array}$ & $\begin{array}{l}\text { List of random } \\
\text { numbers }^{\mathrm{a}}\end{array}$ & $n / r$ & $n / r$ & $n / r$ & $n / r$ \\
\hline $\begin{array}{l}\text { Ratanarat } 2012 \\
{[29]}\end{array}$ & $\begin{array}{l}\text { List of random } \\
\text { numbers }\end{array}$ & $n / r$ & $n / r$ & $n / r$ & $n / r$ \\
\hline OMAKI 2012 [30] & $\begin{array}{l}\text { Computer } \\
\text { generated }\end{array}$ & $\begin{array}{l}\text { Yes (sequentially numbered sealed } \\
\text { opaque envelopes) }\end{array}$ & No & Yes & Yes - 1/39 CWHD(inclusion mistake) \\
\hline \multicolumn{6}{|l|}{ Crossover } \\
\hline Maher 1988 [31] & $n / r$ & $n / r$ & $n / r$ & $n / r$ & $n / r$ \\
\hline Alarabi 1992 [32] & $n / r$ & $n / r$ & $n / r$ & $\mathrm{n} / \mathrm{r}$ & $n / r$ \\
\hline Jeffery 1994 [33] & $\mathrm{n} / \mathrm{r}$ & $n / r$ & $n / r$ & $\mathrm{n} / \mathrm{r}$ & $n / r$ \\
\hline Kellum 1998 [34] & $n / r$ & $n / r$ & $n / r$ & $n / r$ & Yes - $2 \mathrm{CWH}$ and $1 \mathrm{CWHD/13}$ died prior to crossover \\
\hline $\begin{array}{l}\text { Garcia-Fernandez } \\
2000[35]\end{array}$ & $n / r$ & $n / r$ & No & $n / r$ & $n / r$ \\
\hline Maxvold $2000[36]$ & $n / r$ & $n / r$ & $n / r$ & $n / r$ & $n / r$ \\
\hline Wynkel 2004 [37] & $n / r$ & $n / r$ & $n / r$ & $n / r$ & $n / r$ \\
\hline Ricci 2006 [38] & $n / r$ & $n / r$ & $n / r$ & $\mathrm{n} / \mathrm{r}$ & $n / r$ \\
\hline Davies 2008 [39] & $\mathrm{n} / \mathrm{r}$ & $n / r$ & $n / r$ & $n / r$ & Yes - 14/45 not crossed over \\
\hline
\end{tabular}

abtained after author contact. Abbreviations: $\mathrm{CVVH}$, continuous venovenous hemofiltration; CVVHD, continuous venovenous hemodialysis; IHD, intermittent hemodialysis; n/r, not reported. 
Pooled data from two small crossover trials using similar dose CVVH vs. CVVHD $[34,38]$ suggest that hemofiltration may shorten the time to filter failure, although only the RoM result achieved statistical significance (MD -7.3 hours, $95 \%$ CI -19.4 to $+4.9, P=0.24, I^{2}=38 \%$; RoM 0.67, $95 \%$ CI 0.45 to $\left.0.99, P=0.04, I^{2}=7 \% ; \mathrm{n}=50\right)$. Incorporating the results of one parallel-group trial using similar dose CVVH vs. hemodiafiltration (CVVHDF) [27] produced a pooled result that was significant for both effect measures (MD -5.4 hours, $95 \%$ CI -9.6 to -1.3 hours, $P=$ 0.01; RoM 0.70, 95\% CI 0.56 to $0.88, P=0.003 ; I^{2}=0 \%$ in both analyses; $\mathrm{n}=113$ ). Recognizing that higher dose may also affect filter life, two other trials comparing nonequivalent doses in the two treatment arms demonstrated shorter time to filter clotting in the higher dose group. One crossover trial comparing higher dose CVVH to CVVHDF [39] demonstrated an even greater decrease in filter life in the CVVH group, and one parallel group trial comparing lower dose CVVH to CVVHDF [26] demonstrated a non-statistically significant shorter time to filter clotting in the higher-dose group (Figure 3). Including the data from trials with non-equivalent doses in the two treatment arms in the pooled analysis resulted in a similar shortened time to filter failure in the hemofiltration group (MD -5.6 hours, $95 \%$ CI -10.4 to -0.9 hours, $P=0.02$; RoM $0.69,95 \%$ CI 0.50 to $0.95, P=0.02$; five trials, 383 patients), with higher heterogeneity $\left(I^{2}=51\right.$ to $\left.66 \%\right)$. All these trials used unfractionated heparin anti-coagulation and pre-filter replacement fluid except for one trial that used a mixture of pre- and post-filter replacement to keep the filtration fraction $<20 \%$ [38]. This reduction in filter survival time of about one-third is equivalent to a $50 \%$ increase in filters required for hemofiltration compared to hemodialysis. 


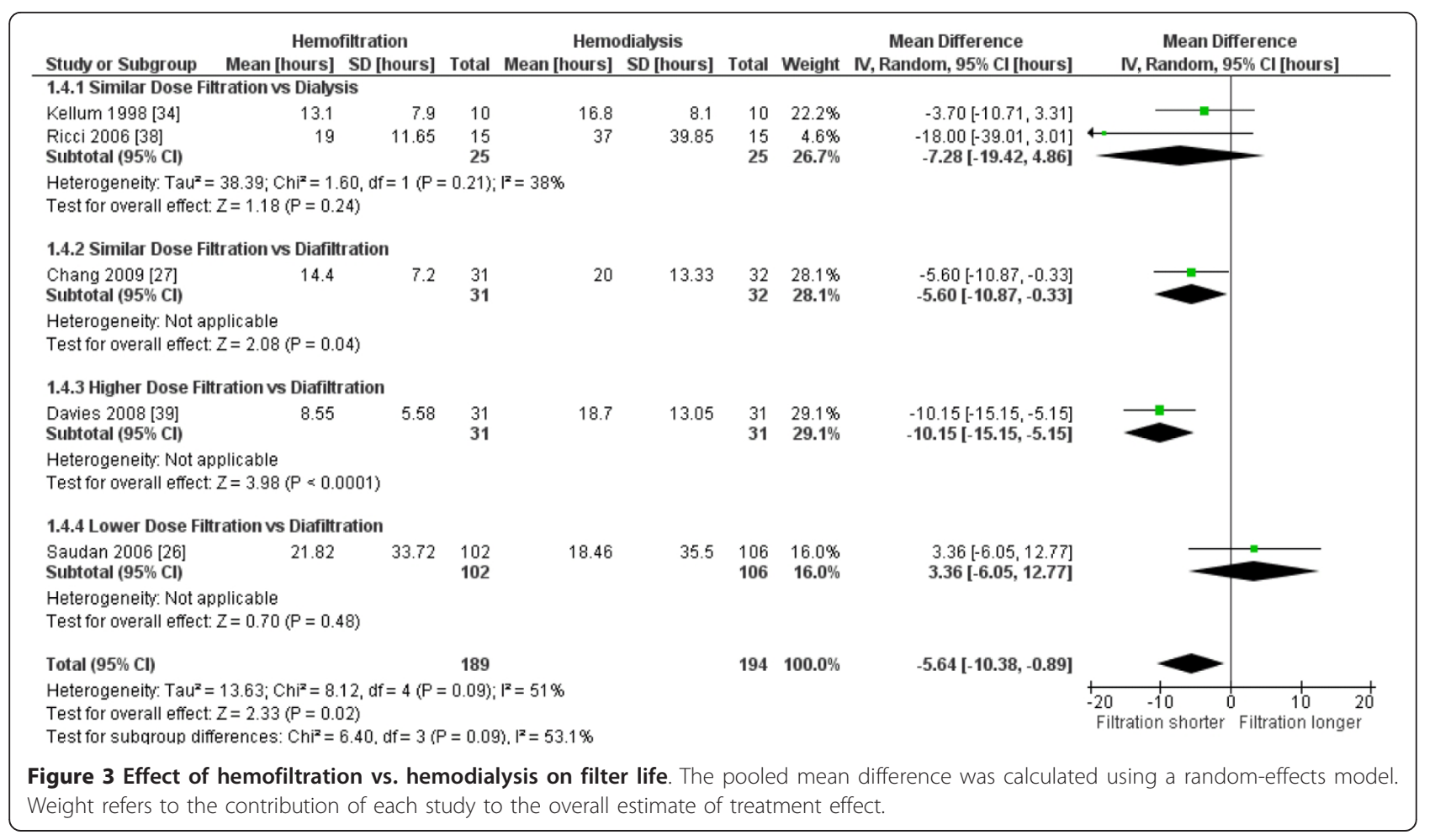

\section{Clearances of small and large molecules (Table 4)}

Few molecules were examined in more than one study, and analyses included few patients. In general, small molecule clearance (for example, urea, phosphate and creatinine) was similar between hemofiltration and hemodialysis, whereas hemofiltration achieved higher clearance of larger molecules (up to around 20 kiloDaltons $(\mathrm{kDa}))$. Pooled data from two studies [24,34] showed similar clearance of interleukin (IL)- 6 between modes, but statistical heterogeneity was high. Single studies also found that hemofiltration delivered significantly higher clearances of protein and albumin (using a high (60 kDa) cut-off filter) [24], and almost all amino acids in one study examining pediatric patients receiving amino acid supplementation [36].

The impact of clearance mode on serum concentrations of various solutes of interest was reported even less frequently. One crossover trial [34] found the concentration of tumor necrosis factor (TNF) $\alpha$ (but not IL-6, IL-10, SL-selectin, and endotoxin) to be significantly lower in the patients during hemofiltration. A second crossover trial [35] found no differences in concentrations of mediators of endothelial activation. One parallel-group trial [25] measured a larger decrease in IL-6 and smaller increase in TNF $\alpha$ concentration in the hemofiltration group, but these cytokines were only measured in one patient treated with hemofiltration and two patients treated with hemodialysis. Finally, the crossover trial of pediatric patients receiving amino acid supplementation [36] reported lower serum concentrations of amino acids in association with higher clearances in the hemofiltration group.

\section{Discussion}

This systematic review and meta-analysis highlights the paucity of data from randomized controlled trials comparing hemofiltration to hemodialysis in the treatment of AKI. Considering clinical outcomes of hemofiltration in parallel-group RCTs, there was no indication of improved mortality or organ dysfunction, although confidence intervals were wide. Our meta-analysis suggests that hemofiltration shortens filter life by about five to six hours (or one-third of total mean filter time). Based primarily on crossover RCTs, we found that hemofiltration increases the clearance of medium to larger molecules compared to hemodialysis. Almost no studies determined whether the enhanced middle-molecule clearance attributed to hemofiltration actually led to lower serum concentrations.

The trials reporting on filter failure rates used primarily pre-filter addition of replacement fluid. Comparing pre-filter to post-filter addition, one trial [37] included in this meta-analysis and other studies [67-69] have demonstrated that replacement fluid requirements are 
Table 4 Clearance measurements of hemofiltration vs.

\begin{tabular}{|c|c|c|c|c|c|}
\hline \multirow[b]{2}{*}{ Molecular substance } & \multirow[b]{2}{*}{$\begin{array}{l}\text { Number of trials; number of patients } \\
\text { randomized }\end{array}$} & \multicolumn{4}{|c|}{ Change in clearance hemofiltration vs hemodialysis ${ }^{\mathrm{a}}$} \\
\hline & & $\begin{array}{c}\text { Effect } \\
\text { estimate }\end{array}$ & $\begin{array}{l}95 \% \text { confidence } \\
\text { interval }\end{array}$ & $\begin{array}{c}P- \\
\text { value }\end{array}$ & $\begin{array}{l}\text { Heterogeneity } \\
\left(I^{2}\right)\end{array}$ \\
\hline \multicolumn{6}{|l|}{ Smaller molecules } \\
\hline Urea (60 Da) & $4[33,36-38] ; 49$ & $+1 \%^{\mathrm{b}}$ & $-2 \%$ to $+3 \%$ & 0.60 & $0 \%$ \\
\hline Phosphate (95 Da) & $1[37] ; 18$ & $0 \%^{c}$ & $-4 \%$ to $+4 \%$ & 1.00 & $\mathrm{n} / \mathrm{a}$ \\
\hline Creatinine (113 Da) & $3[33,37,38] ; 43$ & $+1.8 \%^{\mathrm{b}}$ & $-0.4 \%$ to $+4.1 \%$ & 0.12 & $0 \%$ \\
\hline Uric acid (168 Da) & $2[33,37] ; 28$ & $+4 \%$ & $+1 \%$ to $+7 \%$ & 0.01 & $0 \%$ \\
\hline \multicolumn{6}{|l|}{ Larger molecules } \\
\hline Vancomycin (1.8 kDa) & $1[33] ; 10$ & $+18 \%$ & $+8 \%$ to $+28 \%$ & 0.0003 & $\mathrm{n} / \mathrm{a}$ \\
\hline$\beta_{2}$-microglobulin (11.8 kDa) & $2[37,38] ; 33$ & $+94 \%^{\mathrm{d}}$ & $+78 \%$ to $+112 \%$ & $<0.0001$ & $0 \%$ \\
\hline $\begin{array}{l}\text { IL-1 Receptor Agonist (16-18 } \\
\mathrm{kDa} \text { ) }\end{array}$ & $1[24] ; 12$ & $+77 \% \%^{e, f}$ & $+24 \%$ to $+153 \%$ & 0.002 & $n / a$ \\
\hline $\begin{array}{l}\text { Retinol Binding Protein }(21.2 \\
\text { kDa) }\end{array}$ & 1 [37]; 18 & $+42 \%$ & $+4 \%$ to $+94 \%$ & 0.03 & $\mathrm{n} / \mathrm{a}$ \\
\hline IL-6 (26 kDa) & $2[24,34] ; 22$ & $+6 \%{ }^{f, g}$ & $-62 \%$ to $+191 \%$ & 0.91 & $89 \%$ \\
\hline \multicolumn{6}{|c|}{ 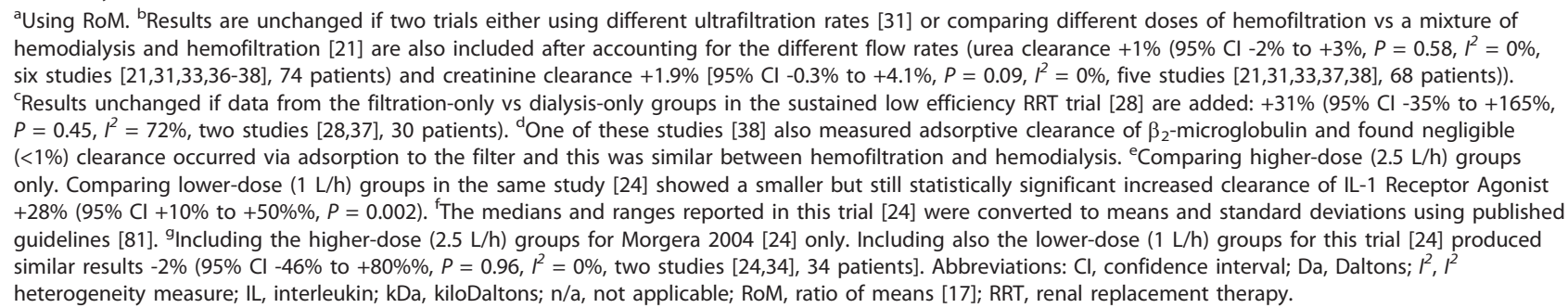 } \\
\hline
\end{tabular}

about 15 to $20 \%$ higher to achieve similar small molecule clearance rates at doses of around $20 \mathrm{~mL} / \mathrm{kg} / \mathrm{h}$. Our finding of a $33 \%$ shorter time to filter failure with hemofiltration, combined with its higher fluid requirements to achieve similar small molecule clearance, implies that hemofiltration may consume more resources than hemodialysis. This hypothesis merits further evaluation in a formal cost analysis.

Comparing the published practice survey data in different countries and regions [10-15] (summarized in Figure 4), it appears that physicians tend to use a mode with at least some hemofiltration (that is, either CVVH or CVVHDF), perhaps anticipating additional benefit associated with hemofiltration. However, the small number of trials, randomized patients and events does not support this belief. A well-designed and adequately powered trial would be necessary to establish the superiority of hemofiltration.

Strengths of our review include methods to minimize bias, such as a comprehensive literature search, duplicate data abstraction, consideration of important clinical outcomes, and inclusion of additional methodological or clinical information from authors. The primary limitation is the small number and size of RCTs comparing pure hemofiltration to pure hemodialysis at similar doses. Sensitivity analyses, including trials whose arms also varied with respect to dose, gave similar results, as expected given recent large trials $[4,5]$ and meta-analyses [6,7] that found similar outcomes with different RRT doses. In addition, trials varied in the modality of RRT used, timing of initiation, and types of filters and blood flows, although recent meta-analyses have not found differential outcomes based on these factors [70-73]. We did not consider comparisons of blood clearance modes with peritoneal dialysis $[74,75]$, which is used in some areas of the world to treat acute kidney failure. Finally, heterogeneity may have been underestimated because these tests are underpowered when there are few trials. Although hemofiltration is of particular interest in patients with sepsis, in whom proinflammatory mediators are increased, there was insufficient data to conduct a subgroup analysis in these patients. In addition, we did not examine the role of hemofiltration vs hemodialysis in patients with sepsis who have not yet developed AKI [40] or the role of hemofiltration compared to no RRT [76-79].

\section{Conclusions}

Pooled data from a few randomized trials suggest that hemofiltration increases the clearance of medium to larger molecules without improving clinical outcomes, though confidence intervals are wide. Hemofiltration may also reduce filter life. This latter finding, together 


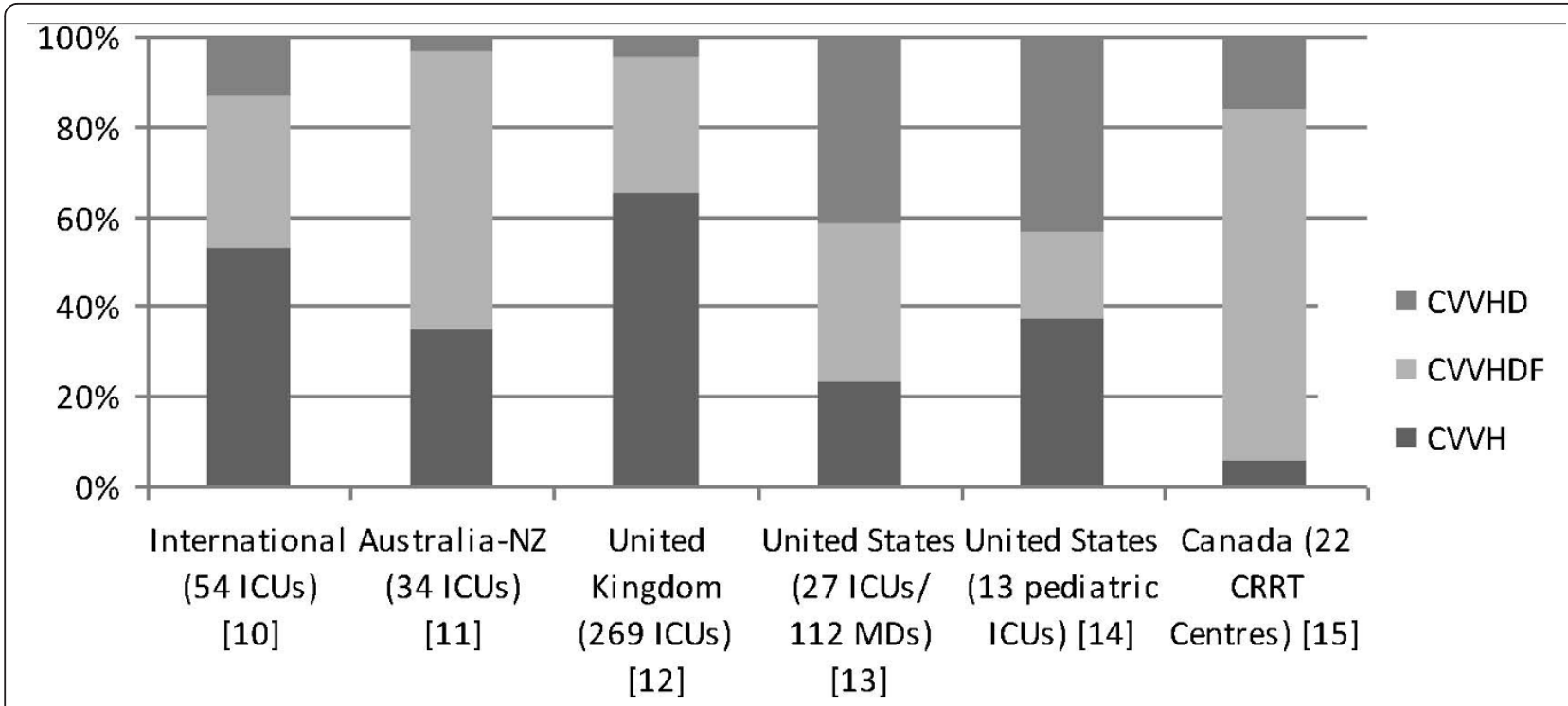

Figure 4 Distribution of mode of RRT used in different countries/regions based on practice surveys. Abbreviations: CRRT, continuous renal replacement therapy; CWH, continuous venovenous hemofiltration; CWHD, continuous venovenous hemodialysis; CWHDF, continuous venovenous hemodiafiltration; ICU, intensive care unit; MD, medical doctor; NZ, New Zealand; RRT, renal replacement therapy; UK, United Kingdom; US, United States.

with the increased replacement fluid requirements to achieve equivalent small-molecule clearance when prefilter replacement is used, suggests that hemofiltration may be more expensive than hemodialysis. Our findings support the need for additional pilot data [80] to evaluate the impact of hemofiltration vs. hemodialysis on intermediate outcomes, such as vasopressor requirements, that may serve as valid surrogates for important clinical outcomes that could subsequently be evaluated in a large definitive trial.

\section{Key messages}

- Few randomized controlled trials have compared hemofiltration vs hemodialysis for the treatment of acute kidney injury.

- Pooling the results from these trials does not suggest beneficial clinical outcomes of hemofiltration vs hemodialysis, but confidence intervals are wide.

- Compared to hemodialysis, hemofiltration may increase clearance of medium to larger molecules, but may also shorten the time to filter failure.

- Additional pilot trials are needed to evaluate the impact of hemofiltration vs. hemodialysis on intermediate outcomes, such as vasopressor requirements, that may serve as valid surrogates for important clinical outcomes that could subsequently be evaluated in a large definitive trial.

\section{Abbreviations}

AKI: acute kidney injury; APACHE: acute physiology and chronic health evaluation; Cl: confidence interval; CRRT: continuous renal replacement therapy; CWH: continuous veno-venous hemofiltration; CWHD: continuous veno-venous hemodialysis; CWHDF, continuous veno-venoous hemodiafiltraion; HD: hemodialysis; HF: hemofiltration; $P^{2}: P$ heterogeneity measure; ICU: intensive care unit; IL: interleukin; kDa: kiloDalton; MD: mean difference; MODS: multi-organ dysfunction score; RCT: randomized controlled trial; RoM: ratio of means; RR: relative risk; RRT: renal replacement therapy; SAPS: simplified acute physiology score; SOFA: sequential organ functional assessment; TNF: tumor necrosis factor

\section{Acknowledgements}

We are grateful to Eric Alarmartine [22], Jai Won Chang [27], Kamaliah Bt. Mohd Daud [25], Andrew Davenport [21], Yong Li [40], Stanislao Morgera [24], Ville Pettila [23], Ranistha Ratanarat [28], Claudio Ronco [41,62] and Patrick Saudan [26] for providing additional data from their trials or for informing us that no additional data were available. We also thank Jennifer Tsang for assistance with translation of Chinese articles [40,53-55]. We appreciate the thoughtful advice of Michelle Hladunewich, David Klein, Stephen Lapinsky and Robert Richardson from the Toronto Acute Kidney Injury Research Group. This study received no specific funding. Jan Friedrich and Karen Burns hold Clinician Scientist Awards with the Canadian Institutes of Health Research (CIHR). Sean Bagshaw holds a Canada Research Chair in Critical Care Nephrology and a Clinician Scientist Award from Alberta Innovates - Health Solutions (AI-HS). Neither CIHR nor Al-HS had any involvement in the design and conduct of the study.

\section{Author details}

${ }^{1}$ Department of Medicine, University of Toronto, Toronto, ON, M5G 2C4, Canada. ${ }^{2}$ Department of Medicine, St. Michael's Hospital, 30 Bond Street, Toronto, ON M5B 1W8, Canada. ${ }^{3}$ Critical Care Department, St. Michael's Hospital, 30 Bond Street, Toronto, ON M5B 1W8, Canada. ${ }^{4}$ The Keenan Research Centre in the Li Ka Shing Knowledge Institute, St. Michael's Hospital, Toronto, ON M5B 1W8, Canada. ${ }^{5}$ Interdepartmental Division of Critical Care, University of Toronto, Toronto, ON, M5B 1W8, Canada. ${ }^{6}$ Division of Critical Care Medicine, Faculty of Medicine and Dentistry, University of Alberta, Edmonton, AB T6G 2B7, Canada. ${ }^{7}$ Department of Critical Care Medicine and Sunnybrook Research Institute, Sunnybrook Health Sciences Centre, 2075 Bayview Avenue, Toronto, ON M4N 3M5, Canada.

\section{Authors' contributions}

JF and NA acquired and analyzed the data. JF wrote the first draft of the manuscript. All authors were involved with the conception and design of 
the study, interpretation of the data, and critical revision of the manuscript for important intellectual content. All authors have read and approved the manuscript for publication.

\section{Competing interests}

The authors declare that they have no competing interests.

Received: 10 May 2012 Revised: 17 July 2012 Accepted: 6 August 2012 Published: 6 August 2012

\section{References}

1. Uchino S, Kellum J, Bellomo R, Doig GS, Morimatsu H, Morgera S, Schetz M, Tan I, Bouman C, Macedo E, Gibney N, Tolwani A, Ronco C, for the Beginning and Ending Supportive Therapy for the Kidney (BEST Kidney) Investigators: Acute Renal Failure in Critically III Patients: A multinational, multicenter study. JAMA 2005, 294:813-818.

2. Bagshaw SM, Lapinsky S, Dial S, Arabi Y, Dodek P, Wood G, Ellis P Guzman J, Marshall J, Parrillo JE, Skrobik Y, Kumar A, the Cooperative Antimicrobial Therapy of Septic Shock (CATSS) Database Research Group: Acute kidney injury in septic shock: clinical outcomes and impact of duration of hypotension prior to initiation of antimicrobial therapy. Intensive Care Med 2009, 35:871-881.

3. Wald R, Quinn RR, Luo J, Li P, Scales DC, Mamdani MM, Ray JG, for the University of Toronto Acute Kidney Injury Research Group: Chronic dialysis and death among survivors of acute kidney injury requiring dialysis. JAMA 2009, 302:1179-1185.

4. The RENAL Replacement Therapy Study Investigators: Intensity of continuous renal-replacement therapy in critically ill patients. N Engl J Med 2009, 361:1627-1638.

5. The VA/NIH Acute Renal Failure Trial Network: Intensity of renal support in critically ill patients with acute kidney injury. N Eng J Med 2008, 359:7-20.

6. Van Wert R, Friedrich JO, Scales DC, Wald R, Adhikari NKJ: High-dose renal replacement therapy for acute kidney injury: systematic review and meta-analysis. Crit Care Med 2010, 38:1360-1369, addendum Crit Care Med 2010, 38:2424-2425.

7. Jun M, Heerspink HJL, Ninomiya T, Gallagher M, Bellomo R, Myburgh J, Finfer S, Palevsky PM, Kellum JA, Perkovic V, Cass A: Intensities of renal replacement therapy in acute kidney injury: a systematic review and meta-analysis. Clin J Am Soc Nephrol 2010, 5:956-963.

8. Brunet S, Leblanc M, Geadah D, Parent D, Courteau S, Cardinal J: Diffusive and convective solute clearances during continuous renal replacement therapy at various dialysate and ultrafiltration flow rates. Am J Kidney Dis 1999, 34:486-492.

9. Ronco C, Tetta C, Mariano F, Wratten ML, Bonello M, Bordoni V, Cardona X, Inguaggiato $P$, Pilotto $L$, d'Intini $V$, Bellomo R: Interpreting the mechanisms of continuous renal replacement therapy in sepsis: the peak concentration hypothesis. Artif Organs 2003, 27:792-801.

10. Uchino S, Bellomo R, Morimatsu H, Morgera S, Schetz M, Tan I, Bouman C, Macedo E, Gibney N, Tolwani A, Oudemans-van Straaten H, Ronco C, Kellum JA: Continuous renal replacement therapy: a worldwide practice survey. The beginning and ending supportive therapy for the kidney (B. E.S.T. kidney) investigators. Intensive Care Med 2007, 33:1563-1570.

11. Renal Study Investigators: Renal replacement therapy for acute kidney injury in Australian and New Zealand intensive care units: a practice survey. Crit Care Resusc 2008, 10:225-230.

12. Gatward JJ, Gibbon GJ, Wrathall G, Padkin A: Renal replacement therapy for acute renal failure: a survey of practice in adult intensive care units in the United Kingdom. Anaesthesia 2008, 63:959-966.

13. Overberger P, Pesacreta M, Palevsky PM, for the VA/NIH Acute Renal Failure Trial Network: Management of Renal Replacement Therapy in Acute Kidney Injury: a survey of practitioner prescribing practices. Clin J Am Soc Nephrol 2007, 2:623-630.

14. Flores FX, Brophy PD, Symons JM, Fortenberry JD, Chua AN, Alexander SR, Mahan JD, Bunchman TE, Blowey D, Somers MJG, Baum M, Hackbarth R, Chand D, McBryde K, Benfield M, Goldstein SL: Continuous renal replacement therapy (CRRT) after stem cell transplantation. A report from the prospective pediatric CRRT Registry Group. Pediatr Nephrol 2008, 23:625-630.

15. Zwillman M, Adhikari NK, Meade MO, Hladunewich M, Piquette D, Friedrich JO: Survey of continuous renal replacement therapy in Canadian intensive care units [Abstract]. Inten Care Med 2006, 32:S161.
16. Mills EJ, Chan A-W, Wu P, Vail A, Guyatt GH, Altman DG: Design, analysis, and presentation of crossover trials. Trials 2009, 10:27.

17. Friedrich $\mathrm{JO}$, Adhikari NK, Beyene J: The ratio of means method as an alternative to mean differences for analyzing continuous outcome variables in meta-analysis: a simulation study. BMC Med Res Methodol 2008, 8:32.

18. Higgins JP, Thompson SG: Quantifying heterogeneity in a meta-analysis. Stat Med 2002, 21:1539-1558.

19. Higgins JPT, Thompson SG, Deeks JJ, Altman DG: Measuring inconsistency in meta-analyses. BMJ 2003, 327:557-560.

20. Elbourne DR, Altman DG, Higgins JPT, Curtin F, Worthington HV, Vail A: Meta-analyses involving cross-over trials: methodological issues. Int J Epidemiol 2002, 31:140-149.

21. Davenport A, Will EJ, Davidson AM: Improved cardiovascular stability during continuous modes of renal replacement therapy in critically ill patients with acute hepatic and renal failure. Crit Care Med 1993, 21:28-338.

22. Alamartine E, de Filippis JP, Toulon J, Berthoux F: On-line continuous venovenous hemodiafiltration: a technique for the control of ultrafiltration and convection during continuous renal replacement therapy. Ren Fail 1994, 16:707-714.

23. Pettila $\mathrm{V}$, Tiula $\mathrm{E}$ : Intermittent hemodiafiltration in acute renal failure in critically ill patients. Clin Nephrol 2001, 56:324-331.

24. Morgera S, Slowinski T, Melzer C, Sobottke V, Vargas-Hein O, Volk T, Zuckermann-Becker H, Wegner B, Müller JM, Baumann G, Kox WJ, Bellomo R, Neumayer $\mathrm{H}-\mathrm{H}$ : Renal replacement therapy with high-cutoff hemofilters: impact of convection and diffusion on cytokine clearances and protein status. Am J Kidney Dis 2004, 43:444-453.

25. Daud KM, Leong GB, Visvanathan R: Acute dialytic support for the critically ill: continuous venovenous haemodialysis versus continuous venovenous haemofiltration. Int Med J 2006, 13:37-42.

26. Saudan P, Niederberger M, De Seigneux S, Romand J, Pugin J, Perneger T, Martin PY: Adding a dialysis dose to continuous hemofiltration increases survival in patients with acute kidney failure. Kidney Int 2006, 70:1312-1317.

27. Chang JW, Lee HK, Yang WS, Kim SB, Park S-K, Lee SK, Park JS: No difference in clearance and survival between continuous hemodiltration and hemodiafiltration at the same net effluent in patients with acute renal failure [Abstract]. Crit Care Med 2009, 37(Suppl):A473.

28. Ratanarat R, Rugsasuk S, Chanchairujira T, Athirakul K, Vasuvattakul S: Effect of prolonged intermittent renal replacement therapy on phosphate kinetics in critically ill patients with acute kidney injury [Abstract]. J Am Soc Nephrol 2009, 20:602A.

29. Ratanarat R, Chaipruckmalakarn T, Laowahutanont N, Larpparisuth N, Vasuvattakul S: Efficacy and hemodynamic outcome of prolonged intermittent renal replacement therapy (PIRRT) in critically ill patients: a preliminary report. J Med Assoc Thai 2012, 95(Suppl 2):S265-S271.

30. Wald R, Friedrich JO, Bagshaw SM, Burns KEA, Garg AX, Hladunewich MA, House AA, Lapinsky S, Pannu NI, Richardson RM, Thorpe K, Adhikairi N: The optimal mode of renal replacement therapy in acute kidney injury (OMAKI): a pilot randomized controlled trial of CVVH vs. CVVHD [Abstract]. J Am Soc Nephrol 2011, 22:10B.

31. Maher ER, Hart L, Levy D, Scoble JE, Baillod RA, Sweny P, Varghese Z, Moorhead JF: Comparison of continuous arteriovenous haemofiltration and haemodialysis in acute renal failure [Letter]. Lancet 1988, 1:129.

32. Alarabi AA, Taube A, Danielson BG, Wikstroem B: Continuous ateriovenous haemodialysis and haemofiltration in intensive care acute renal failure patients. Int Urol Nephrol 1992, 24:657-664.

33. Jeffrey RF, Khan AA, Prabhu P, Todd N, Goutcher E, Will EJ, Davison AM: A comparison of molecular clearance rates during continuous hemofiltration and hemodialysis with a novel volumetric continuous renal replacement system. Artif Organs 1994, 18:425-428.

34. Kellum JA, Johnson JP, Kramer D, Palevsky P, Brady JJ, Pinsky MR: Diffusive vs. convective therapy: effects on mediators of inflammation in patients with severe systemic inflammatory response syndrome. Crit Care Med 1998, 26:1995-2000.

35. Garcia-Fernandez N, Lavilla FJ, Rocha E, Purroy A: Haemostatic changes in systemic inflammatory response syndrome during continuous renal replacement therapy. J Nephrol 2000, 13:282-289.

36. Maxvold NJ, Smoyer WE, Custer JR, Bunchman TE: Amino acid loss and nitrogen balance in critically ill children with acute renal failure: a 
prospective comparison between classic hemofiltration and hemofiltration with dialysis. Crit Care Med 2000, 28:1161-1165.

37. Wynckel A, Cornillet J, Bene B, Stolz A, Lepouse C, Paris B, Chanard J: Improved removal of small proteins using continuous venovenous hemofiltration to treat acute renal failure. ASAIO J 2004, 50:81-84.

38. Ricci Z, Ronco C, Bachetoni A, D'amico G, Rossi S, Alessandri E, Rocco M, Pietropaoli P: Solute removal during continuous renal replacement therapy in critically ill patients: convection versus diffusion. Crit Care 2006, 10:R67.

39. Davies HT, Leslie G, Pereira SM, Webb SA: A randomized comparative crossover study to assess the affect on circuit life of varying pre-dilution volume associated with CVVH and CVVHDF. Int J Artif Organs 2008, 31:221-227.

40. Li Y, Hu ZJ, Li CX, Cui Y, Dai QC, Guo JP: A clinical study on different modalities of blood purification for the treatment of sepsis [Chinese]. Chin Crit Care Med 2009, 21:369-370.

41. Marchionna N, Floris M, Brendolan A, Nalesso F, Zanella M, Ronco C: Solutes removal during continuous renal replacement therapy in critically ill patients with a new high polysulfone-based hemofilter (CUREFLO ${ }^{\circledR}$ ): convection vs. diffusion [Abstract]. J Am Soc Nephrol 2010, 21:893A.

42. Raja R, Karmer M, Goldstein S, Caruana R, Lerner A: Comparison of continuous arteriovenous hemofiltration and continuous arteriovenous dialysis in critically ill patients. ASAIO Trans 1986, 435-436.

43. Zobel G, Ring E, Zobel V: Continuous arteriovenous renal replacement systems for critically ill children. Ped Nephrol 1989, 3:140-143.

44. Bellomo R, Ernest D, Parkin G, Boyce N: Clearance of vancomycin during continuous arteriovenous hemodiafiltration. Crit Care Med 1990, 18:181-183.

45. Hirasawa H, Sugai T, Ohtake Y, Oda S, Shiga H, Matsuda K, Kitamura N Continuous hemofiltration and hemodiafiltration in the management of multiple organ failure. Contrib Nephrol 1991, 93:42-46.

46. Valtonen M, Tiula E, Neuvonen PJ: Effect of continuous venovenous haemofiltration and haemodiafiltration on the elimination of fluconazole in patients with acute renal failure. J Antimicrob Chemother 1997, 40:695-700.

47. Meloni C, Morosetti M, Turani F, Palombo G, Meschini L, Zupancich E, Taccone-Gallucci M, Di Giulio S, Casciani CU: Cardiac function and oxygen balance in septic patients during continuous hemofiltration. Blood Purif 1998, 16:140-146.

48. Booth M, Fox C, Dobb G: Energy losses associated with continuous venovenous haemodiafiltration in intensive care patients [Abstract]. $\mathrm{Br}$ J Anaesth 1995, 74(Suppl 1):109.

49. Favre H, Blanc E, Emonet S, Claeysen D: Treatment of acute renal insufficiency with continuous veno-venous haemofiltration; importance of precise fluid balance control [French] [Abstract]. Schweiz Med Wochenschr 1996, 126(Suppl 81):17.

50. Cole L, Bellomo R, Baldwin I: A randomized, prospective, crossover study of the hemodynamic effects of high-volume hemofiltration in patients with septic shock [Conference Proceedings]. Blood Purif 1998, 16:113-114.

51. Dilek K, Ersoy A, Güllűlű M, Aslanhan I, Emir G, Dalkỷlỷç E, Karabulut Y, Usta M, Ilcol Y, Yavuz M, Yurtkuran M: Crush syndrome developing acute renal failure in the Marmar earthquake: the comparison of the effects of different hemodialysis approaches on renal functions and mortality rates [Abstract]. Nephol Dial Transplant 2000, 15:A82.

52. Lins RL, Elseviers MM, Van der Niepen $P$, Hoste $E$, Malbrain ML, Damas $P$ Devriendt J, SHARF investigators: Intermittent versus continuous renal replacement therapy for acute kidney injury patients admitted to the intensive care unit: results of a randomized clinical trial. Nephol Dial Transplant 2009, 24:512-518.

53. Zhao SP, Wu J, Ai YH, Sun B, Xu DM, Guo QL: Comparison of clinical efficacy between continuous veno-venous hemofiltration (CVVH) and $\mathrm{CVVH}$ combined with hemoperfusion for the treatment of multiple organ dysfunction syndrome [Chinese]. Chin Crit Care Med 2009, 21:373-374.

54. Lui P, He YZ, Zhang XG, Li G, Wang HC, Lu XX, Zhang CG, Meng QY: Studying the therapic effects of hemoperfusion with continuous venovenous hemofiltration on the patients with acute paraquat poisoning] [Chinese]. Chin J Industrial Hygiene Occupat Dis 2011, 29:266-269.
55. Guo JP, Hu ZJ, Li CX, Li Y, Cui Y, Jia CM: Clinical comparison of efficiency in clearance of interleukin-8 and life of filtrator with continuous venous blood filtration using different proportion of pre- and post-dilution of filtration fluid] [Chinese]. Chin Crit Care Med 2011, 23:189-190.

56. Hubsher J, Olshan AR, Schwartz AB, Zoranski B, DeClement F, Bendlin A, Hensell D, Brezin JH, Krevolin LE, Chinitz JL: Continuous arteriovenous hemofiltration for the treatment of anasarca and acute renal failure in severely burned patients. ASAIO Trans 1986, 32:401-404.

57. Schetz M: Removal of cytokines in septic patients using continuous veno-venous hemodiafiltration. Crit Care Med 1994, 22:715-716, author reply $719-721$.

58. Wakabayashi Y, Kamijou Y, Soma K, Ohwada T: Removal of circulating cytokines by continuous haemofiltration in patients with systemic inflammatory response syndrome or multiple organ dysfunction syndrome. Br J Surg 1996, 83:393-394.

59. Hirayama Y, Hirasawa H, Oda S, Shiga H, Nakanishi K, Matsuda K, Nakamura M, Hirano T, Moriguchi T, Watanabe E, Nitta M, Abe R, Nakada T: The change in renal replacement therapy on acute renal failure in a general intensive care unit in a university hospital and its clinical efficacy: a Japanese experience. Ther Apher Dial 2003, 7:475-482.

60. Davenport A, Will EJ, Davison AM: Effect of renal replacement therapy on patients with combined acute renal and fulminant hepatic failure. Kidney Int Suppl 1993, 43:S245-S251.

61. Davenport A, Will EJ, Davison AM: Continuous vs. intermittent forms of haemofiltration and/or dialysis in the management acute renal failure in patients with defective cerebral autoregulation at risk of cerebral oedema. Contrib Nephrol 1991, 93:225-233.

62. Floris M, Marchionna N, Clementi A, Kim JC, Cruz DN, Nalesso F, Zanella M, Garzotto F, de Cal M, Virzì GM, Brendolan A, Ronco C: Evaluation of a new polysulfone hemofilter for continuous renal replacement therapy. Blood Purif 2011, 32:133-138.

63. Knaus WA, Draper EA, Wagner DP, Zimmermann JE: APACHE II: a severity of disease classification system. Crit Care Med 1985, 13:818-829.

64. Le Gall JR, Lemeshow S, Saulnier F: A new Simplified Acute Physiology Score (SAPS II) based on a European/North American multicenter study. JAMA 1993, 270:2957-2963, [Erratum, JAMA 1994, 271:1321.]

65. Ferreira FL, Bota DP, Bross A, Melot C, Vincent JL: Serial evaluation of the SOFA score to predict outcome in critically ill patients. JAMA 2001, 286:1754-1758.

66. Marshall JC, Cook DJ, Christou NV, Bernard GR, Sprung CL, Sibbald WJ: Multiple organ dysfunction score: a reliable descriptor of a complex clinical outcome. Crit Care Med 1995, 23:1638-1652.

67. Troyanov S, Cardinal J, Geadah D, Parent D, Courteau S, Caron S, Leblanc M: Solute clearances during continuous venovenous haemofiltration at various ultrafiltration flow rates using multiflow-100 and HF1000 filters. Nephol Dial Transplant 2003, 18:961-966.

68. Parakininkas D, Greenbaum LA: Comparison of solute clearance in three modes of continuous renal replacement therapy. Pediatr Crit Care Med 2004, 5:269-274.

69. Nurmohamed SA, Jallah BP, Vervloet MG, Beishuizen A, Groeneveld ABJ: Predilution versus postdilution continuous venovenous hemofiltration: no effect on filter life and azotemic control in critically ill patients on heparin. ASAIO J 2011, 57:48-52.

70. Pannu N, Klarenbach S, Wiebe N, Manns B, Tonelli M, Alberta Kidney Disease Network: Renal replacement therapy in patients with acute renal failure: a systematic review. JAMA 2008, 299:793-805.

71. Bagshaw SM, Berthiaume LR, Delaney A, Bellomo R: Continuous versus intermittent renal replacement therapy for critically ill patients with acute kidney injury: a meta-analysis. Crit Care Med 2008, 36:610-617.

72. Seabra VF, Balk EM, Liangos O, Sosa MA, Cendoroglo M, Jaber BL: Timing of renal replacement therapy initiation in acute renal failure: a metaanalysis. Am J Kidney Dis 2008, 52:272-284.

73. Wu M-Y, Hsu Y-H, Bai C-H, Lin Y-F, Wu C-H, Tam K-W: Regional citrate versus heparin anticoagulation for continuous renal replacement therapy: a meta-analysis of randomized controlled trials. Am J Kidney Dis 2012, 59:810-818.

74. Phu NH, Hien TT, Mai NTH, Chau TTH, Chuong LV, Loc PP, Winearls C, Farrar J, White N, Day N: Hemofiltration and peritoneal dialysis in infection-associated acute renal failure in Vietnam. N Engl J Med 2002, 347:895-902. 
75. George J, Varma S, Kumar S, Thomas J, Gopi S, Pisharody R: Comparing continuous venevenous hemodiafiltration and periotoneal dialysis in critically ill patients with acute kidney injury: a pilot study. Perit Dial Int 2011, 31:422-429.

76. Payen D, Mateo J, Cavaillon JM, Fraisse F, Floriot C, Vicaut E, Hemofiltration and Sepsis Group of the College National de Reanimation et de Medecine d'Urgence des Hopitaux extra-Universitaires: Impact of continuous venovenous hemofiltration on organ failure during the early phase of severe sepsis: a randomized controlled trial. Crit Care Med 2009, 37:803-810.

77. Sander A, Armbruster W, Sander B, Daul AE, Lange R, Peters J: Hemofiltration increases IL- 6 clearance in early systemic inflammatory response syndrome but does not alter IL- 6 and TNF alpha plasma concentrations. Intensive Care Med 1997, 23:878-884.

78. Laurent I, Adrie C, Vinsonneau C, Cariou A, Chiche JD, Ohanessian A, Spaulding C, Carli P, Dhainaut JF, Monchi M: High-volume hemofiltration after out-of-hospital cardiac arrest: a randomized study. J Am Coll Cardiol 2005, 46:432-437.

79. Honore PM, Jamez J, Wauthier M, Lee PA, Dugernier T, Pirenne B, Hanique G, Matson JR: Prospective evaluation of short-term, high-volume isovolemic hemofiltration on the hemodynamic course and outcome in patients with intractable circulatory failure resulting from septic shock. Crit Care Med 2000, 28(11):3581-3587.

80. Arnold DM, Burns KE, Adhikari NK, Kho ME, Meade MO, Cook DJ, McMaster Critical Care Interest Group: The design and interpretation of pilot trials in clinical research in critical care. Crit Care Med 2009, 37(1 Suppl):S69-74.

81. Hozo SP, Djulbegovic B, Hozo I: Estimating the mean and variance from the median, range, and the size of a sample. BMC Med Res Methodol 2005, 5:13.

doi:10.1186/cc11458

Cite this article as: Friedrich et al: Hemofiltration compared to hemodialysis for acute kidney injury: systematic review and meta-analysis. Critical Care 2012 16:R146.

\section{Submit your next manuscript to BioMed Central and take full advantage of:}

- Convenient online submission

- Thorough peer review

- No space constraints or color figure charges

- Immediate publication on acceptance

- Inclusion in PubMed, CAS, Scopus and Google Scholar

- Research which is freely available for redistribution

Submit your manuscript at www.biomedcentral.com/submit 